\title{
LEADING INNOVATIVE TEAMS USING ANP
}

\author{
Sam Sharp \\ Numerix Pty. Ltd. \\ Level 8, 65 York Street \\ Sydney, NSW 2000 Australia \\ E-mail: sam.sharp@numerix.com.au \\ Mark Long \\ Learning and Assessment Services \\ PO Box 241 Alexandria \\ NSW 1435 Australia \\ E-mail:malong@aapt.net.au
}

\begin{abstract}
'Agile' project management has provided the main paradigm for managing software projects over the past decade, recognizing the inherent uncertainty and volatility in many areas of the work. It is natural to attempt the extension of these methodologies to general knowledge-work projects, i.e. those typically requiring specialist skills and expertise. One of the most appealing characteristics of the approach is the empowerment of teams, which are frequently vested with intellectual autonomy and decision-making responsibility.

In this paper we show how modern teams working in these areas can take carriage of the innovation process. We argue that the worth of an innovative idea can only be evaluated rationally if its connection to the ultimate objective is explicit and if it can be properly compared with that of its competitors. Connections are best represented in a network diagram, commonly favored in ideation environments. Nodes here depict not only the solution alternatives and objectives, but drivers, sub-drivers, criteria and other intermediate problem or decision entities through which solutions are expected to exercise their effect on the objective.
\end{abstract}

We propose that once idea generation has been exhausted, the network diagram, useful for discussion and testing connections, be converted to an influence matrix which is much more amenable to analysis and evaluation.

From here teams can use AHP/ANP prioritizing techniques to evaluate the relative strength or importance of the influences of outgoing arcs on their immediate neighbors. Conventional ANP methodology can be applied, prioritizing cluster-to-cluster effects to provide a weighted supermatrix and then a limit matrix. 
By providing teams with a natural transition to ANP via the influence matrix, they can make rational and transparent decisions instead of subjecting alternatives to a vote or some sort of ranking system as is the common practice.

Keywords: Innovation, Agile, leadership, ANP

\section{Introduction}

Innovation represents an area of large and increasing importance in both the private and public sectors as organizations search for a competitive edge or improved performance. Much of the literature discusses ideas about creativity, culture, leadership and approaches for promoting innovation. However very little material exists that covers practical decision-making steps which convert ideas and inventions into innovative products.

This paper will show how the ANP/AHP is a logical consequence of a properly executed and documented innovation process, providing as it does the end of a sequence which starts either with the decomposition of problems or decisions into drivers or criteria, or with intuitive idea generation. Either way, a rational approach demands that connections ultimately be made between these entities. This is best represented in the form of a network diagram and then its corresponding directed adjacency or influence matrix which is nothing more than an equally weighted but yet to be normalized supermatrix. Full and proper prioritization using AHP/ANP techniques translates this finally into a mechanism by which optimum innovative decisions can be identified.

Our motivation here is to introduce AHP/ANP as a natural technique for innovative teams to clarify preferences and priorities at all stages of the innovation process. In particular, we believe that the while the early creative phases, characterized by brainstorming and other techniques is necessarily divergent, in a world of constraints the onus falls on leaders to help teams converge toward consensus. We believe that the prioritization of the various influences leaving nodes relative to some control criterion provides a significant means for doing this, allowing remaining sharp differences to be highlighted and then reconciled through a transparent process of discussion, justification, weighting and geometric averaging.

\section{Literature Review}

The two books listed below are good examples of some excellent material being presented about the innovative environment and the many meta-issues which affect it. However, the reader is left grasping for tangible and practical tools with which to implement some of these. There is no shortage of sources that are similar in this respect. 
- Adaptive Leadership - Accelerating Enterprise Agility, Jim Highsmith, AddisonWesley (2014)

- The Australian Leadership Paradox, Geoff Aigner and Liz Skelton, Allen \& Unwin, (2013)

\section{Hypotheses/Objectives}

It is our hypothesis that innovation leaders lack concrete and practical tools which can allow the fruits of creative thinking and idea generation to be transformed into solutions via a sound and rigorous decision-making model.

Our objectives are:

1. To enable innovation practitioners to move beyond innovation philosophy and toward real and practical tools which lead to resolution backed by rigor, transparency and team effort.

2. To show that full and free brainstorming sessions are best documented by means of an influence diagram which not only provides clarity of cause and effect, but also acts as a rather natural precursor to decision resolution via the ANP supermatrix which emerges once prioritizations of influences have been introduced.

3. To provide leaders, particularly those responsible for innovative teams, the tools with which to provide constraining guidance toward consensus once the radical and divergent thinking that characterizes the ideation phase has run its course. We claim that the very practice of prioritization lends a sense of convergence to the team and where it does not, highlights points of disagreement which can then be the focus of greater and more detailed resolution provided that this benefits the overall initiative.

\section{Research Design/Methodology}

In this paper we are not concerned with a specific model but rather a general process which incorporates both well-established ideation techniques such as brainstorming and sound decision processes such as the ANP. We claim that because the process is based upon cause and effect relationships, it is sufficiently general to support problem-solving or decision-making exercises and that these relationships, once prioritized for strength or importance, provides the means for valid judgment and selection via ANP. We have had the opportunity to test these ideas with innovative groups such as scientists and construction designers and are able to report favorable results. 


\section{Data/Model Analysis}

As stated earlier, we are not presenting a particular model here but do aim to show examples drawn from our experience.

\section{Limitations}

While we suspect our approach to be fully general, this cannot be proven. We can, however, assemble our experience and document successes which would provide plausible support for our claims. We believe that the limitations of the method are to be found in the large number of comparisons that will be required, a problem encountered by many of our colleagues in the ANP community. We aim to incorporate some of the solutions to this problem in evidence in the literature. We believe also that the difficulty of finding solution application software that can be used by teams to implement these methods might be an obstacle. We are attempting to address this.

\section{Conclusions}

Our conclusions from experience gained thus far is that the approach has been received enthusiastically by innovative teams but that its sustained use by those teams is limited by the practical considerations mentioned in Question 6 above.

We believe that our contribution stands apart from many others in the innovation community in that it offers practical steps and tools as opposed to the commonly delivered philosophical messages about the need for culture change and the importance of innovation.

\section{Key References}

Saaty, T.L., \& Peniwati, K. (2007). Group decision-making: Drawing out and reconciling differences. Pittsburgh, PA: RWS Publications.

Saaty, Thomas L. Principia Mathematica Decernendi - (Mathematical Principles of Decision-Making), (2010)

\section{Appendices}

